CERN-TH 6471

HU-TFT 92-15

\title{
INDEX THEOREMS AND LOOP SPACE GEOMETRY
}

\author{
A. Hietamäki \\ Research Institute for Theoretical Physics, University of Helsinki \\ Siltavuorenpenger 20 C, SF-00170 Helsinki, Finland \\ and
}

Antti J. Niemi *

Theory Division, CERN, CH-1211 Geneva 23, Switzerland ${ }^{\dagger}$

\begin{abstract}
We investigate the evaluation of the Dirac index using symplectic geometry in the loop space of the corresponding supersymmetric quantum mechanical model. In particular, we find that if we impose a simple first class constraint, we can evaluate the Callias index of an odd dimensional Dirac operator directly from the quantum mechanical model which yields the Atiyah-Singer index of an even dimensional Dirac operator in one more dimension. The effective action obtained by BRST quantization of this constrained system can be interpreted in terms of loop space symplectic geometry, and the corresponding path integral for the index can be evaluated exactly using the recently developed localization techniques.
\end{abstract}

\footnotetext{
* E-mail: ANIEMI@PHCU.HELSINKI.FI

$\dagger$ permanent address: Research Institute for Theoretical Physics, University of Helsinki, Siltavuorenpenger 20 C, SF-00170 Helsinki, Finland

April 1992

CERN-TH 6471

HU-TFT 92-15
} 
1. Introduction. There are many different ways to characterize supersymmetric theories. Maybe the best known is the existence of a Nicolai transformation [1]. It identifies the fermion determinant of a supersymmetric path integral as a Jacobian determinant for a change of variables that takes the bosonic action into a Gaussian form.

Recently a conceptually different, geometric characterization of supersymmetry has been proposed [2]. This approach originates from the ideas introduced in [3], [4], and is based on the formalism developed in [5], [6]. It relates a supersymmetric theory to symplectic geometry in a superloop space, with half of the bosonic and fermionic variables viewed as superloop space coordinates, and the remaining half of these variables viewed as superloop space one-forms. In this superloop space the action of a supersymmetric theory is locally exact with respect to a model independent superloop space equivariant exterior derivative: The noninteracting part of the action is a linear combination of two terms, a loop space symplectic two-form and the contraction of the corresponding symplectic one-form with a model independent supervector field, that determines a natural action of the group $\mathrm{SO}(2)$. The interaction part of the action can then be identified as a locally exact one-form in the superloop space. This characterization of supersymmetry in terms of (super)symplectic geometry appears to be at least as general as the characterization in terms of a Nicolai transformation. Indeed, in this approach the Nicolai transformation can be viewed as a loop space change of variables to (locally defined) Darboux variables.

In [3] the results of [4] are applied to evaluate the Atiyah-Singer index of a Dirac operator on a compact manifold: The supersymmetric quantum mechanical path integral which yields the index, localizes to the critical points of the action corresponding to constant field configurations. The correct expression for the index then follows from the infinite dimensional Duistermaat-Heckman integration formula [4]. In [5] these observations are combined with the localization technique developed in [6], to evaluate the Atiyah-Singer index of a Dirac operator on a compact manifold and with arbitrary background gauge fields. When combined with the formalism developed in [2], these observations then suggest a potentially useful method for evaluating path integrals in generic supersymmetric theories. In the present paper we shall be interested in developing such methods: As a concrete example we shall consider path integrals which are relevant for the computation of the Callias index [7] of odd-dimensional Dirac operators. We explain how the localization technique introduced in $[5,6]$ generalizes to this case. Furthermore, in addition we find that the Callias index of an odd dimensional Dirac operator can also be obtained from the quantum mechanical path integral that yields the Atiyah-Singer index of a related Dirac operator in one more dimensions. This connection between the index formulas in even and odd dimensions emerges, when we consider the even dimensional quantum mechanical model together with a constraint that effectively eliminates one space dimension. The BRST quantization of the ensuing constrained system yields an effective action, which admits an interpretation in terms of loop space symplectic geometry. The evaluation of the corresponding path integral using the localization technique introduced in $[5,6]$ then yields the correct expression 
for the index in odd dimensions.

2. $D=1$ Index Theorem: We shall first illuminate our general approach by investigating the evaluation of the index of the one-dimensional Dirac hamiltonian

$$
\mathcal{H}=i \sigma^{1} \frac{d}{d q}+\sigma^{2} W_{q}=\left(\begin{array}{cc}
0 & D \\
D^{\dagger} & 0
\end{array}\right)
$$

Here

$$
D=i \frac{d}{d q}-i W_{q}(q)
$$

and the derivative of the superpotential $W(q)$ has a soliton profile,

$$
W_{q}( \pm \infty)=a_{ \pm}
$$

The corresponding supersymmetric quantum mechanical model is obtained by identifying the operators $D$ and $D^{\dagger}$ as generators of a canonical supersymmetry algebra,

$$
\begin{aligned}
& \left(\begin{array}{cc}
0 & D \\
0 & 0
\end{array}\right) \rightarrow Q=\frac{1}{\sqrt{2}} \eta\left(p+i W_{q}\right) \\
& \left(\begin{array}{cc}
0 & 0 \\
D^{\dagger} & 0
\end{array}\right) \rightarrow Q^{\dagger}=\frac{1}{\sqrt{2}} \bar{\eta}\left(p-i W_{q}\right)
\end{aligned}
$$

where $\eta$ and $\bar{\eta}$ are anticommuting variables, and the nontrivial Poisson brackets are

$$
\{p, q\}=\{\bar{\eta}, \eta\}=1
$$

The supersymmetry algebra is

$$
\begin{gathered}
\left\{Q, Q^{\dagger}\right\}=H \\
\{Q, Q\}=\left\{Q^{\dagger}, Q^{\dagger}\right\}=\{Q, H\}=\left\{Q^{\dagger}, H\right\}=0
\end{gathered}
$$

with

$$
H=\left\{Q, Q^{\dagger}\right\}=\frac{1}{2} p^{2}+\frac{1}{2} W_{q}^{2}+i \bar{\eta} W_{q q} \eta
$$

The index of the operator (1) is obtained in the $T \rightarrow \infty$ limit of the superpartition function

$$
Z=\operatorname{Tr}\left\{e^{i T H}\right\}=\int[d p d q][d \bar{\eta} d \eta] \exp \left\{i S_{B}+i S_{F}\right\}
$$

with

$$
S_{B}+S_{F}=\int_{0}^{T} p \dot{q}+\bar{\eta} \dot{\eta}-\left\{Q, Q^{\dagger}\right\}
$$

In order interpret (8), (9) in terms of superloop space symplectic geometry, we introduce the canonical transformation

$$
p \rightarrow e^{-\Phi} p e^{\Phi}=p+\{p, \Phi\}+\frac{1}{2}\{\{p, \Phi\}, \Phi\}+\ldots
$$




$$
q \rightarrow e^{-\Phi} q e^{\Phi}=q+\{q, \Phi\}+\frac{1}{2}\{\{q, \Phi\}, \Phi\}+\ldots
$$

If we select

$$
\Phi(q)=-i \int^{q} W\left(q^{\prime}\right) d q^{\prime}
$$

we get

$$
\begin{gathered}
p \stackrel{\Phi}{\longrightarrow} p-i W(q) \\
q \stackrel{\Phi}{\longrightarrow} q
\end{gathered}
$$

For the generators of the supersymmetry algebra this yields

$$
\begin{gathered}
Q \stackrel{\Phi}{\longrightarrow} e^{-\Phi} Q e^{\Phi}=\frac{1}{\sqrt{2}} \eta p \\
Q^{\dagger} \stackrel{\Phi}{\longrightarrow} e^{-\Phi} Q^{\dagger} e^{\Phi}=\frac{1}{\sqrt{2}} \bar{\eta}(p-2 i W(q))
\end{gathered}
$$

and for the action

$$
S_{B}+S_{F} \rightarrow \int_{0}^{T} p \dot{q}+\bar{\eta} \dot{\eta}-\frac{1}{2} p^{2}+i p W_{q}(q)+i \bar{\eta} W_{q q}(q) \eta
$$

Defining

$$
\begin{gathered}
\eta \rightarrow \frac{1}{\sqrt{2}}\left(\theta_{1}+i \theta_{2}\right) \\
\bar{\eta} \rightarrow \frac{1}{\sqrt{2}}\left(\theta_{1}-i \theta_{2}\right) \\
p \rightarrow-i p+\dot{q}
\end{gathered}
$$

we then get

$$
S_{B}+S_{F}=\int_{0}^{T} \frac{1}{2} \dot{q}^{2}+\frac{1}{2} p^{2}-p W_{q}+\frac{1}{2}\left(\theta_{1} \dot{\theta}_{1}+\theta_{2} \dot{\theta}_{2}\right)-\theta_{2} W_{q q} \theta_{1}
$$

In this form the action can be directly interpreted in terms of superloop space symplectic geometry. For this we consider a superloop space with $q(t)$ and $\theta_{2}(t)$ viewed as superloop space coordinates, and $\theta_{1}(t)$ and $p(t)$ as one-forms. The exterior derivative is

$$
\mathbf{d}=\theta_{1} \frac{\delta}{\delta q}+p \frac{\delta}{\delta \theta_{2}}
$$

and if we introduce the superloop space symplectic one-form

$$
\vartheta=-\frac{1}{2} \dot{q} \theta_{1}+\frac{1}{2} \theta_{2} p
$$


and the corresponding symplectic two-form which determines superloop space Poisson brackets is the exterior derivative of (18),

$$
\Omega=\mathbf{d} \vartheta=\frac{1}{2} p^{2}+\frac{1}{2} \theta_{1} \dot{\theta}_{1}
$$

and if we define the vector field

$$
\mathbf{i}_{S}=-\dot{q} \cdot \mathbf{i}_{\theta_{1}}-\dot{\theta}_{2} \cdot \mathbf{i}_{p}
$$

we get the noninteracting part of the supersymmetric quantum mechanics action (16) as

$$
S=\left(\mathbf{d}+\mathbf{i}_{S}\right) \vartheta=\Omega+\mathbf{i}_{S} \vartheta=\int_{0}^{T} \frac{1}{2} \dot{q}^{2}+\frac{1}{2} p^{2}+\frac{1}{2} \theta_{1} \dot{\theta}_{1}+\frac{1}{2} \theta_{2} \dot{\theta}_{2}
$$

The interaction is obtained by defining the superloop space scalar i.e. zero-form

$$
\mathcal{W}=\theta_{2} W_{q}
$$

Since the interior multiplication of a loop space vector field and a loop space scalar vanishes,

$$
\mathbf{i}_{S} \mathcal{W}=0
$$

we then find that in the superloop space the action (16) can be represented as $(p \rightarrow-p)$

$$
S_{B}+S_{F}=\left(\mathbf{d}+\mathbf{i}_{S}\right)(\vartheta+\mathcal{W})=\int_{0}^{T} \frac{1}{2} \dot{q}^{2}+\frac{1}{2} p^{2}+p W_{q}+\frac{1}{2}\left(\theta_{1} \dot{\theta}_{1}+\theta_{2} \dot{\theta}_{2}\right)+\theta_{1} W_{q q} \theta_{2}
$$

Notice in particular, that this action is a linear combination of exact forms with degree zero, one and two.

We shall now proceed to the evaluation of the path integral (8), (24) using the localization techniques developed in $[5,6]$. For this we first observe that for the superloop space Lie derivative,

$$
\mathcal{L}_{S}=\mathbf{d i}_{S}+\mathbf{i}_{S} \mathbf{d}=-\dot{q} \partial_{q}-\dot{\theta}_{2} \partial_{\theta_{2}}-\ddot{p} \mathbf{i}_{p}-\dot{\theta}_{1} \mathbf{i}_{\theta_{1}}=-\partial_{t}
$$

Obviously

$$
\mathcal{L}_{S}\left(\dot{q} \theta_{1}\right)=0
$$

Hence we conclude from $[5,6]$ that the path integral (8), (24) remains intact if we redefine円

$$
\vartheta \rightarrow \vartheta_{\beta}=-\frac{\beta}{2} \dot{q} \theta_{1}+\frac{1}{2} \theta_{2} p
$$

\footnotetext{
${ }^{1}$ Notice that as explained in [5], we can not redefine the second term in (18) in the same manner. Due to the constant mode, such a redefinition is not a small, local variation.
} 
For the action this yields

$$
S_{B}+S_{F} \rightarrow\left(\mathbf{d}+\mathbf{i}_{S}\right)\left(\vartheta_{\beta}+\mathcal{W}\right)=\int_{0}^{T} \frac{\beta}{2} \dot{q}^{2}+\frac{1}{2} p^{2}+p W_{q}+\frac{\beta}{2} \theta_{1} \dot{\theta}_{1}+\frac{1}{2} \theta_{2} \dot{\theta}_{2}+\theta_{1} W_{q q} \theta_{2}
$$

and from the arguments presented in [6] we conclude that the path integral with (28) is $\beta$-independent.

We define the path integral measure in (8) as [5]

$$
[d q]\left[d \theta_{1}\right][d p]\left[d \theta_{2}\right]=d q_{0} d \theta_{10} d p_{0} d \theta_{20} \prod_{t} d q_{t} d \theta_{1 t} d p_{t} d \theta_{2 t}
$$

Here we have defined

$$
\begin{array}{ll}
q(t)=q_{0}+q_{t} & \theta_{1}(t)=\theta_{10}+\theta_{1 t} \\
p(t)=p_{0}+p_{t} & \theta_{2}(t)=\theta_{20}+\theta_{2 t}
\end{array}
$$

with $q_{0}, \theta_{10}, p_{0}, \theta_{20}$ the constant modes. We then introduce the change of variables

$$
\begin{gathered}
q(t) \rightarrow q_{0}+\frac{1}{\sqrt{\beta}} q_{t} \\
\theta_{1}(t) \rightarrow \theta_{10}+\frac{1}{\sqrt{\beta}} \theta_{1 t}
\end{gathered}
$$

The pertinent Jacobian in the path integral measure (29) is trivial. Since the path integral is independent of $\beta$ we can take the $\beta \rightarrow \infty$ limit which yields for the action in $(28)$

$S_{B}+S_{F} \rightarrow \int_{0}^{T} \frac{1}{2} \dot{q}_{t}^{2}+\frac{1}{2} p_{0}^{2}+\frac{1}{2} p_{t}^{2}+\frac{1}{2} \theta_{1 t} \dot{\theta}_{1 t}+\frac{1}{2} \theta_{2 t} \dot{\theta}_{2 t}+\theta_{10} W_{q q}\left(q_{0}\right) \theta_{20}+p_{0} W_{q}\left(q_{0}\right)+\mathcal{O}\left(\frac{1}{\sqrt{\beta}}\right)$

Integrating over $\theta_{1}, \theta_{2}$ and $p$ we then get for the path integral

$$
\begin{gathered}
Z=\sqrt{\frac{T}{2 \pi}} \int_{-\infty}^{\infty} d q_{0} d \theta_{10} d \theta_{20} \exp \left\{-\frac{i}{2} T W_{q}^{2}\left(q_{0}\right)+i T \theta_{10} W_{q q}\left(q_{0}\right) \theta_{20}\right\} \\
=\frac{1}{2 \pi} \int_{-\infty}^{\infty} d q_{0} W_{q q}\left(q_{0}\right) \exp \left\{-\frac{i}{2} T W_{q}^{2}\left(q_{0}\right)\right\}
\end{gathered}
$$

and in the $T \rightarrow \infty$ limit this yields for the index of the operator (1)

$$
Z \stackrel{T \rightarrow \infty}{\longrightarrow} \operatorname{Index}(\mathcal{H})=\operatorname{DimKer}(D)-\operatorname{DimKer}\left(D^{\dagger}\right)=\frac{1}{2}\left[\frac{a_{+}}{\left|a_{+}\right|}-\frac{a_{-}}{\left|a_{-}\right|}\right]
$$


This result agrees with the exact evaluation in $[7,8]$, and verifies that in the present case the localization technique developed in $[5,6]$ is applicable.

3. Relation to the Atiyah-Singer Index. In [8] it has been shown how the index of an odd dimensional Dirac operator can be related to the index of an operator defined in one more dimensions by an infinite space version of the Atiyah-Patodi-Singer index theorem. Here, we shall obtain a somewhat different connection between even and odd space index theorems: We find that the index of a Dirac operator can be evaluated from a supersymmetric quantum mechanical model which yields the index of a related Dirac operator in one more dimension. This result is a consequence of a very simple constrained quantization procedure: We select the higher dimensional operator in such a way that it coincides with the lower dimensional operator when we demand translation invariance in one space direction. At the level of quantum mechanical path integrals we impose this translation invariance as a first class constraint, and as a consequence we find that the constrained path integral yields the index of the lower dimensional operator. In particular, in this way we can derive the Callias index theorem directly from the Atiyah-Singer index theorem of a higher dimensional Dirac operator.

As an example, we shall evaluate the one dimensional index formula (34) using a supersymmetric path integral which yields the two dimensional Atiyah-Singer index. For this, we consider the following two dimensional Dirac operator

$$
\mathcal{D}=i \gamma^{i} \partial_{i}-\gamma^{i} A_{i}
$$

Here we use the Pauli-matrix representation $\gamma^{1}=\sigma_{y}$ and $\gamma^{2}=\sigma_{x}$ of the $\gamma$-matrices. Notice that if we set $A_{1}=0$ and demand that $A_{2}$ depends only on the variable $q_{1} \sim q$, the $q_{2}$-derivative becomes quite irrelevant. By ignoring this derivative and identifying $A_{2} \sim W_{q}$ the operator (35) then essentially reduces to the operator (1). We shall now demonstrate, that if we implement these conditions as a canonical first class constraint in the pertinent supersymmetric quantum mechanical model, the path integral evaluation of the Atiyah-Singer index for (35) yields the Callias index (34) of the one dimensional operator (1).

The canonical supersymmetry generator corresponding to (35) is

$$
Q=\frac{1}{\sqrt{2}} \psi^{i}\left(p_{i}-A_{i}\right)
$$

and the Poisson bracket supersymmetry algebra is

$$
\{Q, Q\}=H=\frac{1}{2}\left(p_{i}-A_{i}\right)^{2}+\frac{1}{2} \psi^{i} F_{i j} \psi^{j}
$$

In [5] it has been shown that the index of (35) can be obtained by applying the localization technique to the canonical path integral with action

$$
S=\int_{0}^{T} p_{i} \dot{q}_{i}+\frac{1}{2} \psi_{i} \dot{\psi}_{i}-\frac{1}{2}\left(p_{i}-A_{i}\right)^{2}-\frac{1}{2} \psi^{i} F_{i j} \psi^{j}
$$


In order for this to yield the index of the operator (1), we introduce in (38) the abelian first class constraint

$$
p_{2} \sim 0
$$

If the gauge fields $A_{i}$ are functions of $q_{1}$ only, this constraint commutes with the supersymmetry generator (36),

$$
\left\{p_{2}, Q\right\}=0
$$

Consequently it also commutes with the hamiltonian (37) and we have a first class constrained system.

We shall enforce the constraint (39) in the path integral using the BRST gauge fixing procedure. For this, following [9] we introduce a pair of canonically conjugated Grassman variables with Poisson brackets

$$
\{\mathcal{P}, \eta\}=\{\overline{\mathcal{P}}, \bar{\eta}\}=-1
$$

and commuting Lagrange multiplier variables with

$$
\{\pi, \lambda\}=-1
$$

The BRST operator corresponding to (39) is then

$$
\Omega=\eta p_{2}+\bar{\eta} \pi
$$

In order to fix the gauge in the path integral, we introduce the gauge fermion

$$
\Psi=\frac{1}{\beta} \overline{\mathcal{P}} q_{2}+\mathcal{P} \lambda
$$

where $\beta$ is a parameter. This yields the BRST gauge-fixed action [9]

$$
S=\int_{0}^{T} p_{i} \dot{q}_{i}+\frac{1}{2} \psi_{i} \dot{\psi}_{i}+\pi \dot{\lambda}+\mathcal{P} \dot{\eta}+\overline{\mathcal{P}} \dot{\bar{\eta}}-\frac{1}{2}\left(p_{i}-A_{i}\right)^{2}-\frac{1}{2} \psi^{i} F_{i j} \psi^{j}-\frac{1}{\beta} \eta \overline{\mathcal{P}}-\bar{\eta} \mathcal{P}-\lambda p_{2}-\frac{1}{\beta} \pi q_{2}
$$

We change the variables $\pi \rightarrow \beta \pi$ and $\overline{\mathcal{P}} \rightarrow \beta \overline{\mathcal{P}}$. The corresponding Jacobian is trivial, and since the path integral is $\beta$ independent [9] we can take the $\beta \rightarrow \infty$ limit. In this limit we obtain for the action (45)

$$
S \rightarrow \int_{0}^{T} p_{i} \dot{q}_{i}+\frac{1}{2} \psi_{i} \dot{\psi}_{i}-\frac{1}{2}\left(p_{i}-A_{i}\right)^{2}-\frac{1}{2} \psi_{i} F_{i j} \psi_{j}+\lambda p_{2}+\pi q_{2}
$$

Eliminating $p_{i}, \pi$ we get further

$$
S \rightarrow \int_{0}^{T} \frac{1}{2} \dot{q}_{1}^{2}+\frac{1}{2} \psi_{i} \dot{\psi}_{i}+\frac{1}{2} \lambda^{2}+\dot{q}_{1} A_{1}+\lambda A_{2}-\frac{1}{2} \psi_{i} F_{i j} \psi_{j}
$$


and the pertinent path integral is subject to the condition $q_{2}=0$, which is enforced by a $\delta$-functional.

The gauge fixed action (47) admits an interpretation in terms of loop space equivariant cohomology in a superloop space with coordinates $q_{1}$ and $\psi_{2}$, and with $\psi_{1}$ and $\lambda$ the corresponding superloop space one-forms. For this we define the superloop space exterior derivative

$$
\mathbf{d}=\psi_{1} \partial_{q_{1}}+\lambda \partial_{\psi_{2}}
$$

and vector field

$$
\mathbf{i}_{S}=-\dot{q}_{1} \mathbf{i}_{\psi_{1}}-\dot{\psi}_{2} \mathbf{i}_{\lambda}
$$

The corresponding Lie-derivative is

$$
\mathcal{L}_{S}=\mathbf{d i}_{S}+\mathbf{i}_{S} \mathbf{d}=-\partial_{t}
$$

Defining the one-form

$$
\vartheta=-\frac{1}{2} \dot{q}_{1} \psi_{1}+\frac{1}{2} \psi_{2} \lambda-A_{1} \psi_{1}
$$

and the zero-form

$$
\mathcal{W}=A_{2} \psi_{2}
$$

we then find that the action (47) can be represented as

$$
S=\left(\mathbf{d}+\mathbf{i}_{S}\right)(\vartheta+\mathcal{W})
$$

We shall now proceed to evaluate the corresponding path integral using the localization technique developed in $[5,6]$. For this we first re-define the one-form (51) into

$$
\vartheta \rightarrow-\frac{\beta}{2} \dot{q}_{1} \psi_{1}+\frac{1}{2} \psi_{2} \lambda-A_{1} \psi_{1}
$$

According to $[5,6]$ the path integral is independent of $\beta$. Proceeding as in [5] and (28)-(33) we then find in the $\beta \rightarrow \infty$ limit for the action

$$
\begin{gathered}
i S \rightarrow i \int_{0}^{T} \frac{1}{2} \dot{q}_{1 t}^{2}+\frac{1}{2} \psi_{i t} \dot{\psi}_{i t}+\frac{1}{2} \lambda_{0}^{2}+\frac{1}{2} \lambda_{t}^{2}+\lambda_{0} A_{2}\left(q_{10}\right)-\frac{1}{2} \psi_{i 0} F_{i j}\left(q_{10}\right) \psi_{j 0}+\mathcal{O}\left(\frac{1}{\sqrt{\beta}}\right) \\
\rightarrow-\frac{i}{2} T A_{2}^{2}\left(q_{10}\right)-\frac{i}{2} T \psi_{i 0} F_{i j}\left(q_{10}\right) \psi_{j 0}
\end{gathered}
$$

where we have again decomposed the variables in their constant and non-constant modes, used the $\delta$-function in the functional integral to eliminate the variable $q_{2}$, and in the final step eliminated the nonconstant modes.

The evaluation of the remaining $\psi_{i 0}$ and $q_{10}$ integrals proceeds as in the case of the two-dimensional Atiyah-Singer index for (35) [5]. Indeed, by identifying $A_{2} \sim W_{q}$ and $A_{1} \sim 0$ we conclude that the actions in (33.a) and (55.b) coincide, hence we find again the result (34). Consequently we have found a connection between the two dimensional Atiyah-Singer index of (35) and the one dimensional Callias index of (1): The path 
integral evaluation of the former yields the latter if we impose translation invariance in the $q_{2}$ direction as an abelian first-class constraint in the supersymmetric model (38).

4. Index Theorem in Higher Dimensions: We shall now proceed to generalize the previous results for Dirac operators defined in general D-dimensional (flat) spaces. For this, we first consider the supersymmetry generators discussed in [10],

$$
\begin{aligned}
Q & =\frac{1}{2}\left(\theta_{i} p_{i}+\bar{\theta}_{i} W_{i}\right) \\
\bar{Q} & =\frac{1}{2}\left(\bar{\theta}_{i} p_{i}-\theta_{i} W_{i}\right)
\end{aligned}
$$

where the Poisson brackets of the anticommuting variables are

$$
\left\{\theta_{i}, \theta_{j}\right\}=\left\{\bar{\theta}_{i}, \bar{\theta}_{j}\right\}=\delta_{i j}
$$

and the supersymmetry algebra is

$$
\begin{gathered}
\{Q, Q\}=\{\bar{Q}, \bar{Q}\}=H=\frac{1}{2} p_{i}^{2}+\frac{1}{2} W_{i}^{2}+\bar{\theta}_{i} \theta_{j} W_{i j} \\
\{Q, \bar{Q}\}=\{Q, H\}=\{\bar{Q}, H\}=0
\end{gathered}
$$

As explained in [10], this supersymmetry algebra is relevant to a class of Dirac operators whose index has been considered in [7]. The corresponding canonical supersymmetry action is

$$
S=\int_{0}^{T} p_{i} \dot{q}_{i}+\frac{1}{2} \theta_{i} \dot{\theta}_{i}+\frac{1}{2} \bar{\theta}_{i} \dot{\bar{\theta}}_{i}-\frac{1}{2} p_{i}^{2}-\frac{1}{2} W_{i}^{2}-\bar{\theta}_{i} \theta_{k} W_{i k}
$$

In order to interpret (59) in terms of symplectic geometry in a superloop space we again introduce a canonical conjugation such that

$$
\begin{gathered}
p_{i} \rightarrow p_{i}-i W_{i} \\
q_{i} \rightarrow q_{i}
\end{gathered}
$$

Generalizing (10)-(16) we then find for the action

$$
S \rightarrow \int_{0}^{T} \frac{1}{2} \dot{q}_{i}^{2}+\frac{1}{2} \theta_{i} \dot{\theta}_{i}+\frac{1}{2} \bar{\theta}_{i} \dot{\bar{\theta}}_{i}+\frac{1}{2} p_{i}^{2}-p_{i} W_{i}-\bar{\theta}_{i} W_{i k} \theta_{k}
$$

which generalizes (16) to D dimensions. The corresponding superloop space interpretation is obtained by introducing the equivariant exterior derivative

$$
\mathbf{d}+\mathbf{i}_{S}=\theta_{i} \partial_{q_{i}}-p_{i} \partial_{\bar{\theta}_{i}}-\dot{q}_{i} \mathbf{i}_{\theta_{i}}+\dot{\bar{\theta}}_{i} \mathbf{i}_{p_{i}}
$$

With the one-form

$$
\vartheta=-\frac{1}{2} \dot{q}_{i} \theta_{i}-\frac{1}{2} \bar{\theta}_{i} p_{i}
$$


and zero-form

$$
\mathcal{W}=\bar{\theta}_{i} W_{i}
$$

we can then write the action (61) as

$$
S=\left(\mathbf{d}+\mathbf{i}_{S}\right)(\vartheta+\mathcal{W})
$$

The evaluation of the path integral proceeds as before: We introduce a generalization of (27) to localize the path integral to constant configurations, and repeating the steps that led to (33) we then find that the path integral reduces to finite dimensional integral over the bosonic zero mode

$$
\begin{aligned}
Z & =\int \prod_{i} d q_{i 0} d \bar{\theta}_{i 0} d \theta_{i 0} \exp \left\{-\frac{i}{2} T W_{i}^{2}\left(q_{i 0}\right)-i T \bar{\theta}_{i 0} W_{i k}\left(q_{i 0}\right) \theta_{k 0}\right\} \\
& =\sqrt{\left(\frac{T}{2 \pi}\right)^{n}} \int \prod_{i} d q_{i 0} \operatorname{det}\left\|W_{i j}\left(q_{i 0}\right)\right\| \exp \left\{-\frac{i}{2} T W_{i}^{2}\left(q_{i 0}\right)\right\}
\end{aligned}
$$

Notice that this is a direct generalization of (33) to $\mathrm{D}$ dimensions. The result equals that obtained in [10], and yields in the $T \rightarrow \infty$ limit the index of the corresponding Dirac operator.

5. Relation to the Atiyah-Singer Index. In analogy with (35)-(55) we can relate (66) to the Atiyah-Singer index of a higher dimensional Dirac operator. For this, we need to select the higher dimensional operator in such a manner that it reduces to the one corresponding to (56) if we impose translation invariance in some of the coordinate directions. The ensuing constrained path integral quantization of the higher dimensional supersymmetry action then yields the index the original Dirac operator, obtained in the $T \rightarrow \infty$ limit of (66). As an example we shall here apply the path integral evaluation of the four dimensional Atiyah-Singer index theorem described in [5] to compute the index of a three dimensional Dirac hamiltonian coupled to a $\mathrm{SU}(2)$ Yang-Mills-Higgs system,

$$
\mathcal{H}=\alpha^{k}\left(i \partial_{k}+A_{k}\right)-\beta \Phi
$$

where $A_{k}=A_{k}^{\alpha} T^{\alpha}$ is a hermitean gauge field in an isospin-J representation of $\mathrm{SU}(2)$,

$$
\left[T^{\alpha}, T^{\beta}\right]=i \epsilon^{\alpha \beta \gamma} T^{\gamma} \quad \& \quad \operatorname{Tr}\left\{T^{\alpha} T^{\beta}\right\}=J(J+1) \delta^{\alpha \beta}
$$

and we choose $T^{3}$ to be diagonal with eigenvalues $J, J-1, \ldots,-J$.

For a monopole background the gauge field $A_{k}$ can be viewed as a compact perturbation. As a consequence in the index computation it is usually customary to consider only the Higgs field background [7]. With $\Phi^{a} \sim W_{i}$ the ensuing canonical supersymmetry algebra is then of the form (56), and the index can be evaluated as explained above. Here we shall proceed to describe, how this index can also be obtained from the path integral evaluation of the four dimensional Atiyah-Singer index for the Dirac operator

$$
D=\gamma^{\mu}\left(i \partial_{\mu}-A_{\mu}\right)
$$


If we identify $A_{0} \sim \Phi$ in (67) and require translation invariance in the $\mu=0$ direction, (69) essentially reduces to (67). Consequently, we expect that if we impose translation invariance as a first class constraint in the canonical supersymmetry algebra corresponding to (69), the path integral evaluation of the Atiyah-Singer index for (69) subject to this constraint yields the index of (67). For this, we introduce the four dimensional canonical supersymmetry algebra corresponding to (69) with

$$
Q=\frac{1}{\sqrt{2}} \psi_{\mu}\left(p_{\mu}-A_{\mu}^{\alpha} T^{\alpha}(\varphi)\right)
$$

Here we have realized the gauge generators $T^{\alpha}$ canonically on a co-adjoint orbit: With $\varphi^{a}$ coordinates on the co-adjoint orbit and $\omega_{a b}(\varphi)$ the symplectic two-form corresponding to the given representation of the gauge group, we represent the Lie algebra canonically, in terms of the co-adjoint orbit Poisson brackets

$$
\left\{T^{\alpha}(\varphi), T^{\beta}(\varphi)\right\}=\partial_{a} T^{\alpha} \omega^{a b} \partial_{b} T^{\beta}=\epsilon^{\alpha \beta \gamma} T^{\gamma}(\varphi)
$$

In order for (70) to represent the operator (67) we demand that it is subject to the first class constraint

$$
p_{0} \sim 0
$$

The condition

$$
\left\{p_{0}, Q\right\}=0
$$

then implies that $A_{\mu}$ is independent of the coordinate $q_{0}$, and if we identify $A_{0} \sim \Phi$ we obtain the desired constrained canonical realization of the operator (67). The corresponding supersymmetry algebra is determined by

$$
\{Q, Q\}=H=\frac{1}{2}\left(p_{\mu}-A_{\mu}^{\alpha} T^{\alpha}\right)^{2}+\frac{1}{2} \psi_{\mu} F_{\mu \nu}^{\alpha} T^{\alpha} \psi_{\nu}
$$

and the action which will yield the index of (67) is

$$
S=\int_{0}^{T} p_{\mu} \dot{q}_{\mu}+\theta_{a} \dot{\varphi}^{a}+\frac{1}{2} \psi_{\mu} \dot{\psi}_{\mu}-\frac{1}{2}\left(p_{\mu}-A_{\mu}^{\alpha} T^{\alpha}\right)^{2}-\frac{1}{2} \psi_{\mu} F_{\mu \nu}^{\alpha} T^{\alpha} \psi_{\nu}-\frac{1}{2} c^{a} \omega_{a b} c^{b}
$$

and this action is subject to the constraint (72). Notice that except for the constraint, this action coincides with that used in [5] to evaluate the Atiyah-Singer index of (69). Here $c^{a}$ are anticommuting variables introduced to exponentiate the square-root determinant of the symplectic structure on the co-adjoint orbit, which appears as a measure factor in the canonical path integral [5].

We again enforce the constraint (72) using BRST gauge-fixing. Introducing the corresponding gauge fermion (44) and generalizing the steps (41)-(47) we then find for the action

$$
S \rightarrow \int_{0}^{T}\left\{\frac{1}{2} \dot{q}_{k}^{2}+\frac{1}{2} \psi_{\mu} \dot{\psi}_{\mu}+\frac{1}{2} \lambda^{2}+\theta_{a} \dot{\varphi}^{a}+\dot{q}_{k} A_{k}+\lambda \Phi-\frac{1}{2} \psi_{\mu} F_{\mu \nu}^{\alpha} T^{\alpha} \psi_{\nu}-\frac{1}{2} c^{a} \omega_{a b} c^{b}\right\}
$$


Here $\lambda$ is the Lagrange multiplier field for the constraint (72), and the path integral is subject to the $\delta$-function constraint $q_{0}=0$.

In order to interpret (76) in the superloop space we define

$$
\begin{array}{r}
\mathbf{d}=\psi_{k} \partial_{q_{k}}+\lambda \partial_{\psi_{0}}+c^{a} \partial_{\varphi^{a}} \\
\mathbf{i}_{S}=-\dot{q}_{k} \mathbf{i}_{\psi_{k}}-\dot{\psi}_{0} \dot{i}_{\lambda}-\dot{\varphi}^{a} \mathbf{i}_{c^{a}}
\end{array}
$$

Notice that again we find for the Lie derivative

$$
\mathcal{L}_{S}=\mathbf{d i}_{S}+\mathbf{i}_{S} \mathbf{d}=-\partial_{t}
$$

Defining the one-form

$$
\vartheta=-\frac{1}{2} \dot{q}_{k} \psi_{k}+\frac{1}{2} \psi_{0} \lambda-\theta_{a} c^{a}-A_{k}^{\alpha} T^{\alpha} \psi_{k}
$$

and the zero-form

$$
\mathcal{W}=\psi_{0} A_{0} \sim \psi_{0} \Phi
$$

we then find for the action (76) the representation

$$
S=\left(\mathbf{d}+\mathbf{i}_{S}\right)(\vartheta+\mathcal{W})
$$

We evaluate the path integral using the localization technique developed in $[5,6]$. For this we introduce the following one-parameter generalization of (80),

$$
\vartheta \rightarrow-\frac{\beta}{2} \dot{q}_{k} \psi_{k}+\frac{\beta}{2} \psi_{0} \lambda-\theta_{a} c^{a}-A_{k}^{\alpha} T^{\alpha} \psi_{k}
$$

The general arguments presented in $[5,6]$ again imply that the path integral is independent of the parameter $\beta$. We again divide the variables into their constant and non-constant modes, and using the $\beta$ independence of the path integral we then find in the $\beta \rightarrow \infty$ limit for the action

$$
i S \rightarrow i \int_{0}^{T}\left\{\frac{1}{2} \dot{q}_{k t}^{2}+\frac{1}{2} \psi_{\mu t} \dot{\psi}_{\mu t}+\frac{1}{2} \lambda_{0}^{2}+\theta_{a} \dot{\varphi}^{a}+\lambda_{0} \Phi\left(q_{0}\right)-\frac{1}{2} \psi_{\mu 0} F_{\mu \nu}^{\alpha}\left(q_{0}\right) T^{\alpha} \psi_{\nu 0}-\frac{1}{2} c^{a} \omega_{a b} c^{b}\right\}
$$

and by integrating over the nonconstant modes we finally get

$$
i S \rightarrow \frac{i}{2} T \Phi^{\alpha} \Phi^{\beta} T^{\alpha} T^{\beta}-\frac{i}{2} T \psi_{0 \mu} F_{\mu \nu} \psi_{0 \nu}
$$

Notice that this is a direct generalization of our earlier results (33.a), (55.b) and (66.a). Notice also that this action differs from the corresponding action obtained in the evaluation of the Atiyah-Singer index theorem [5]: The second term in (85) does coincide with that relevant for the computation of the four dimensional Atiyah-Singer index theorem, but the first term in (85) is a consequence of the constraint (72). In the $T \rightarrow \infty$ 
limit only the second term in (85) survives, and we get the index of the operator (68) $[7,8]$

$$
Z \stackrel{T \rightarrow \infty}{\longrightarrow} \frac{1}{8 \pi} J(J+1) \oint d S^{i} B_{i}^{\alpha} \frac{\Phi^{\alpha}}{|\Phi|}
$$

6. In conclusions, we have investigated the localization technique developed in $[5,6]$ for the evaluation of supersymmetric path integrals by computing the index of certain Dirac operators. In each case, the localization technique appears to yield the correct result for the path integral. In particular, we have found that the method also yields a novel point of view to index theorems for odd-dimensional Dirac operators: The index can be computed directly from the path integral relevant to a higher dimensional Atiyah-Singer index, by introducing a simple first class constraint that eliminates the extra dimensions. The ensuing BRST quantized canonical action admits a superloop space interpretation and as a consequence the localization techniques developed in $[5,6]$ become directly applicable.

We thank K. Palo for discussions. 


\section{References}

1. H. Nicolai, Phys. Lett. 89B (1980) 341; Nucl. Phys. B176 (1980) 419

2. A. Yu. Morozov, A.J. Niemi and K. Palo, Physics Letters B271 (1991) 365; Nucl. Phys. B (to appear)

3. M.F. Atiyah, Asterisque 131 (1985) 43; J.-M. Bismut, Comm. Math. Phys. 98 (1985) 213; ibid. 103 (1986) 127

4. J.J. Duistermaat and G.J. Heckman, Inv. Math. 72 (1983) 153; M.F. Atiyah and R. Bott, Topology 23 (1984) 1

5. A. Hietamäki, A.Yu. Morozov, A.J. Niemi and K. Palo, Phys. Lett. B263 (1991) 417

6. M. Blau, E. Keski-Vakkuri and A.J. Niemi, Phys. Lett. B246 (1990) 92; A.J. Niemi and P. Pasanen, Phys. Lett. B253 (1991) 349; E. Keski-Vakkuri, A.J. Niemi, G. Semenoff and O. Tirkkonen, Phys. Rev. D44 (1991) 3899

7. C. Callias, Comm. Math. Phys. 62 (1978) 213; R. Bott and R. Seeley, Comm. Math. Phys. 62 (1978) 235

8. A.J Niemi and G.W. Semenoff, Nucl. Phys. B269 (1986) 131

9. E.S. Fradkin and G.A. Vilkovisky, Phys. Lett. B55 (1975) 224; A.J. Niemi, Phys. Repts. 184 (1989) 147

10. C. Imbimbo and S. Mukhi, Nucl. Phys. B242 (1984) 81 